\title{
Estrategias metodológicas y uso de recursos digitales para la enseñanza de la historia. Análisis de recuerdos y opiniones del profesorado en formación inicial ${ }^{1}$
}

\author{
Cosme J. Gómez Carrasco \\ Universidad de Murcia, Murcia,España; cjgomez@um.es \\ Álvaro Chaparro Sainz \\ Universidad de Málaga, Málaga, España; alvaro.chaparro@uma.es
}

Área de Didáctica de las Ciencias Sociales, Departamento de Didáctica de la Matemática; de las Ciencias Sociales y de las Ciencias Experimentales; Facultad de Ciencias de la Educación; Campus de Teatinos, 29071

María del Mar Felices De la Fuente

Universidad de Almería, Almería, España; marfelices@ual.es

\author{
Ramón Cózar Gutiérrez \\ Universidad de Castilla La Mancha, Albacete, España; Ramon.Cozar@uclm.es
}

\section{RESUMEN}

Los cambios que han acontecido en nuestra sociedad requieren de nuevas estrategias de enseñanza y de nuevas finalidades que construyan una ciudadanía con espíritu crítico y conciencia de pertenencia a un mundo amplio y multicultural. El objetivo principal de esta investigación es analizar los recuerdos del profesorado de Educación Secundaria en formación sobre la metodología didáctica utilizada en las clases de Historia, y sus opiniones sobre las estrategias más adecuadas para enseñar esta materia. La investigación se ha realizado en la especialidad de Geografía e Historia del Máster de Formación del Profesorado. Se seleccionaron cuatro grupos en las universidades de Murcia, Málaga, Castilla-La Mancha y Almería en el curso académico 2018/2019, recogiendo una muestra de 129 participantes $(\mathrm{n}=129)$. Los resultados muestran que no existen grandes diferencias entre universidades tanto en recuerdos como en intenciones sobre la metodología didáctica que creen que deben utilizar en la clase de Historia. Sin embargo, sí hay diferencias significativas de género: las mujeres tienen unos recuerdos más tradicionales de las clases de Historia, y están más a favor del uso de recursos digitales.

Palabras clave: Formación del profesorado; Educación histórica; Metodología didáctica; Recursos digitales; Competencia digital

\section{Methodological strategies and use of digital resources for teaching history. Analysis through the memories and opinions of teachers in initial training}

\begin{abstract}
The changes that have taken place in our society require new teaching strategies and new purposes that build citizenship with a critical spirit and awareness of belonging to a broad and multicultural world. The main objective of this research is to analyze the memories of secondary school teachers in training on the teaching methodology used in history classes, and their opinions on the most appropriate strategies to teach this subject. The research has been carried out in the specialty of Geography and History of the Teacher Training Master. Four groups were selected at the universities of Murcia, Málaga, Castilla-La Mancha and Almería in the 2018/2019 academic year, collecting a sample of 129 participants $(n=129)$. The results show that there are no major differences between universities in both memories and intentions about the teaching methodology that they believe they should use in the History class. However, there are significant gender differences: women have more traditional memories of History classes, and they are more in favor of the use of digital resources.
\end{abstract}

Key words: Teacher education; History education; Teaching methodology; Digital resources; Digital competence

ISSN: 0210-2773

DOI: https://doi.org/10.17811/rifie.49.1.2020.65-74

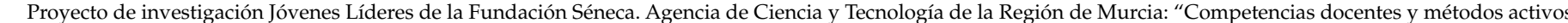

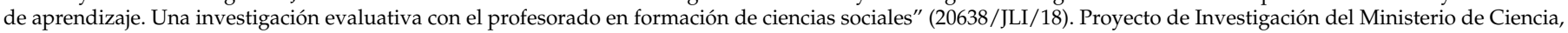

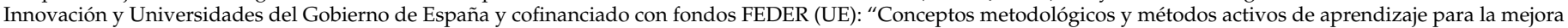
de las competencias docentes del profesorado" (PGC2018-094491-B-C33). 


\section{Introducción}

Los cambios que han acontecido en nuestra sociedad requieren de nuevas estrategias de enseñanza y de nuevas finalidades que construyan una ciudadanía con espíritu crítico y conciencia de pertenencia a un mundo multicultural (Prats, 2010), capaz de actuar en democracia sin ignorar las controversias (Levstik y Barton, 2001). La enseñanza de la historia debe tener, por tanto, una serie de finalidades que contribuyan adecuadamente a la formación del pensamiento histórico del alumnado, algo que precisa del trabajo con procedimientos característicos del método histórico, que transciendan la simple memorización de fechas y datos (Sáiz y Gómez, 2016) y movilicen contenidos de segundo orden (VanSledright, 2014).

En los últimos años, las investigaciones en pensamiento histórico han propuesto distintos modelos acerca de los conceptos y competencias que comprende este tipo de pensamiento y cómo desarrollarlo (Gómez y Miralles, 2015). Se insiste en que la formación de la conciencia histórica pasa por transformar las prácticas didácticas que presiden hoy día la enseñanza de la historia, sustituyéndolas por metodologías activas de aprendizaje, basadas en la indagación, el uso de fuentes o el manejo de las TIC.

Investigaciones recientes se han centrado precisamente en analizar las rutinas heredadas y normalizadas en las aulas de Ciencias Sociales, para entender su permanencia en el tiempo, dándole especial importancia a la formación inicial del profesorado (Parra y Fuertes, 2019). Como señalan Lévesque y Zanazanian (2015), en este proceso de cambio y transformación de las prácticas, a través de la formación inicial podemos no solo formar a nuestro alumnado en nuevas metodologías que mejoren la educación histórica, sino también identificar y deconstruir la asimilación que suele establecer el alumnado entre conocimiento histórico y memorización del pasado.

En esta línea, algunos trabajos han estudiado los recuerdos de los maestros en formación sobre las clases de historia, la metodología empleada y sus concepciones epistemológicas sobre la disciplina y los procesos de enseñanza, siendo los resultados muy similares en todos ellos (Suárez, 2012; Sáiz y Muñoz, 2014). Por su parte, Martínez, Souto y Beltrán (2006) hablan de malas prácticas, abuso del libro de texto, lección magistral y poca motivación ante los modelos didácticos de corte positivista. Entre los recuerdos del alumnado destaca también la presencia del examen como instrumento legitimador de un sistema basado en las rutinas y la memorización (Merchán, 2011; Miralles y Gómez, 2016). Este panorama no dista mucho de los resultados aportados por investigaciones más recientes, como la de Gómez, Rodríguez y Mirete (2018), quienes observan dificultades en el alumnado para delimitar epistemológicamente el significativo de la disciplina histórica, su función y sus posibilidades formativas.

\subsection{TIC, mass-media y patrimonio dentro de los recursos de innovación}

Actualmente, existe un consenso en la necesidad de formar a los futuros maestros en el uso de nuevas tecnologías para desarrollar sus competencias digitales, convirtiéndose en uno de los grandes desafíos de los cuerpos docentes (Cabero y Guerra, 2011; Gutiérrez, Palacios y Torrego, 2010).

Es primordial aproximarse a las concepciones del profesorado en activo de las etapas pre-universitarias, ya que se considera clave para poder actuar convenientemente en las etapas formativas superiores (Arancibia y Badia, 2015; Cabezas, Casillas y Pinto, 2014). Sólo conociendo las prácticas actuales, descubriendo las opiniones del profesorado en activo e identificando la realidad de lo que acontece en el aula, se pueden establecer actuaciones concretas que sanen las carencias detectadas. Entre estas lagunas podemos destacar, por ejemplo, la visión de las TIC como un elemento motivacional (Cózar, González-Calero, Villena y Merino, 2019), en contraposición a otros países donde el uso de las tecnologías resulta más procedimental (Miralles, Gómez y Monteagudo, 2019; Miralles, Gómez, Arias y Fontal, 2019).

Los estudios realizados concluyen que una de las mayores dificultades a la hora de explotar estos recursos en el aula se encuentra en el nivel formativo del profesorado (Colomer, Sáiz y Bel, 2018). En España, pese a los avances en su incursión en los contextos educativos, no se ha producido una implementación real, como indican Ramírez y González (2016), consecuencia de las dificultades del profesorado para su aplicación. Un hecho que según De Haro (2009) impide que las TIC se conviertan en un elemento de cambio, de innovación y de ruptura frente a la enseñanza más tradicional. Incluir nuevas tecnologías en el aula no implica un cambio de enseñanza, ni garantiza la introducción de un proceso innovador; el cambio debe venir de los docentes, de sus formas de hacer y de sus modos de evaluar (Marqués, 2013).

En este contexto formativo, el uso de recursos mass-media en las aulas recibe la valoración positiva del alumnado como freno a las tradicionales metodologías de enseñanza basadas en un aprendizaje repetitivo y conceptual. Las investigaciones realizadas valoran este uso en base, principalmente, a dos criterios: motivación y experimentación, lo que aproxima el aprendizaje a una cuestión lúdica, perdiendo la oportunidad, según Miralles, Gómez y Monteagudo (2019), de aprovecharla para incentivar el cambio metodológico que demanda el alumnado.

Al igual que ocurre con las TIC, la educación patrimonial se ha configurado, en los últimos años, como uno de los recursos mejor valorados para aunar en el aula el desarrollo de competencias, la adquisición de contenidos, la aplicación de metodologías activas de enseñanza o el establecimiento de estrategias innovadoras de aprendizaje. La presencia de elementos patrimoniales en los procesos de enseñanza-aprendizaje se ha consolidado, como lo demuestran Fontal e Ibáñez (2017), convirtiéndose en una fuente fundamental para el conocimiento socio-histórico.

La investigación en torno a las percepciones, tanto del alumnado en formación como del profesorado en activo, sobre el uso del patrimonio en los contextos educativos resulta nuevamente insoslayable para mejorar la enseñanza de los contenidos de Ciencias Sociales (Castro y López, 2019; Cuenca, Estepa y Martín, 2016; Estepa, Ávila y Ferreras, 2008; Jiménez, Cuenca y Ferreras, 2010; Miralles, Gómez y Rodríguez, 2017). Los resultados de estas investigaciones muestran cómo, de cara a superar su carácter anecdótico en las aulas (Estepa, 2001), se reclama una formación específica en educación patrimonial y la necesidad de contar con materiales especializados, demandando así un mejor conocimiento de metodologías concretas de enseñanza (Castro y López, 2019) que muestren cómo usar el patrimonio en el contexto escolar.

\subsection{Diferencias sobre metodologías innovadoras por género}

Desde una perspectiva más amplia, si nos centramos en las percepciones de los futuros maestros hacia el uso de metodologías activas e innovadoras en las aulas, observamos resultados diversos y contradictorios cuando se analizan teniendo en cuenta la variable de género. Los estudiantes valoran muy positivamente los nuevos métodos de enseñanza. Así lo demuestran, por ejemplo, los trabajos de Barba, Martínez y Torrego (2012) o Pegalajar y Colmenero (2013) que se han centrado en investigar el impacto del aprendizaje cooperativo, los de Angelini y García-Carbonell, 
(2015) o Blasco-Serrano, Lorenzo y Sarsa (2018) que han indagado en estrategias de simulación, gamificación y en flipped classroom; o el de Cózar, González-Calero, Villena y Merino (2019), que investiga la valoración que hacen los futuros maestros sobre el uso de la realidad virtual en la enseñanza de la historia.

Sin embargo, cuando se establecen diferencias por género, observamos que las percepciones del alumnado varían en algunas investigaciones, siendo la tendencia que el alumnado masculino se sienta más cómodo con estilos de aprendizaje tradicionales, mientras que las mujeres prefieren estilos de aprendizaje más activos, estando dispuestas en mayor medida a formarse en ello (González-Peiteado y Pino-Juste, 2013; 2014).

Por lo que respecta al uso de las TIC, donde contamos con un mayor volumen de investigación, las diferencias por género han sido tradicionalmente más marcadas. Existe un buen número de estudios que han señalado cómo los hombres parecen tener más conocimientos y habilidades respecto al uso y manejo de estos recursos, en detrimento de las mujeres, que muestran una menor confianza e interés por las nuevas tecnologías (Moya, Hernández, Hernández y Cózar, 2011; Cabero, Barroso, Llorente y Yanes, 2016). Pese a que los hombres parecen tener mayores habilidades técnicas, más experiencia y conocimiento en estas tecnologías, las mujeres destacarían por estar más interesadas en usarlas para el aprendizaje y en su propia formación. Según Cózar y Roblizo (2014), las mujeres superarían a los hombres en un mayor uso social de las TIC y en el número de horas que dedican a utilizar el ordenador, hecho que podemos vincular a la búsqueda de un mayor rendimiento académico.

Desde finales del siglo pasado ya hubo algunos autores como Shaw y Marlow (1999), que pusieron de manifiesto una cierta contradicción entre los resultados de las investigaciones centradas en analizar las percepciones y actitudes de hombres y mujeres frente a las TIC. Al inicio de la era digital, algunos investigadores relacionaron estas diferencias con el menor acceso de las mujeres a los ordenadores (Spennemann, 1996), generándose así lo que se ha denominado brecha digital de género (Castaño, Martín y Martínez, 2011). Sin embargo, en la actualidad, esta tendencia parece estar reduciéndose, pues las investigaciones comienzan a evidenciar la ausencia de relación entre el género y el grado de aceptación de las tecnologías (Cózar, Moya, Hernández y Hernández, 2016; Cózar, González-Calero, Villena y Merino, 2019).

\section{Método}

\subsection{Objetivos}

El objetivo principal de esta investigación es analizar los recuerdos del profesorado de Educación Secundaria en formación sobre la metodología didáctica utilizada en las clases de Historia, y sus opiniones sobre las estrategias más adecuadas para enseñar esta materia. Para ello, este objetivo principal se ha dividido en tres objetivos específicos:

OE1: Detallar los recuerdos de los docentes en formación sobre la metodología didáctica utilizada por sus profesores de historia.

OE2: Detallar la opinión de los futuros docentes sobre las estrategias y técnicas más adecuadas para enseñar historia en Educación Secundaria.

OE3: Detallar la opinión de los futuros docentes acerca del uso concreto de recursos digitales para enseñar historia en Educación Secundaria.

\subsection{Participantes}

La investigación se ha realizado en la especialidad de Geografía e Historia del Máster de Formación del Profesorado. Se seleccionaron cuatro grupos en las universidades de Murcia (UMU), Málaga (UMA), Castilla-La Mancha (UCLM) y Almería (UAL) en el curso académico 2018/2019, representando una muestra total de 129 participantes $(n=129)$. El estudio presenta un balance equilibrado por género ( $55 \%$ hombres y $45 \%$ mujeres), donde casi el $80 \%$ de los participantes tienen entre 22 y 26 años. El porcentaje de participación de cada universidad es similar (27\% en la UMU y UMA; $25 \%$ en la UCLM y $20 \%$ en la UAL). En cuanto a la formación anterior, algo más del 55\% son graduados en Historia, un $25 \%$ son graduados en Historia del Arte, y casi un $20 \%$ son graduados en Geografía, Humanidades y otros títulos afines (Ciencias Políticas, Sociología, etc.).

\subsection{Enfoque}

Para esta investigación el diseño escogido fue cuantitativo no experimental a través de un cuestionario con escala Likert (1-5). Los diseños mediante encuesta son muy habituales en el ámbito de la educación ya que son aplicables a múltiples problemas y permiten recoger información sobre un elevado número de variables (Sapsford \& Jupp 2006).

\subsection{Instrumento de recogida de información y procedimiento de análisis}

Los datos utilizados forman parte de un cuestionario titulado "Recuerdos y percepciones de los docentes en formación sobre metodología didáctica y enseñanza de la Historia en Educación Secundaria", elaborado ad hoc, con una escala de valoración cerrada tipo Likert (1-5). Consta de dos bloques temáticos para cuya realización se han tenido en cuenta trabajos como los de Gómez, Rodríguez y Mirete (2018) o Martínez, Souto y Beltrán (2006). El primero, dedicado a los recuerdos del profesorado en formación sobre las clases de historia, la metodología más frecuente, el tipo de recursos utilizados y el papel activo o pasivo del alumnado. El segundo bloque recoge las percepciones del profesorado en formación sobre los métodos, técnicas y recursos más adecuados para la enseñanza de esta materia. La validación del contenido se realizó mediante el procedimiento inter jueces en torno a las categorías de pertinencia y claridad de los ítems del instrumento.

Los datos se codificaron y analizaron en el programa estadístico SPSS v.22.0 para MAC. Antes de proceder al análisis de datos se estimó el grado de fiabilidad y validez del constructo. Para analizar la fiabilidad del cuestionario se empleó el método de consistencia interna basado en el Alfa de Cronbach que permite estimar la fiabilidad de un instrumento de medida compuesto por un conjunto de ítems, de tipo escala Likert, que esperamos midan la misma dimensión teórica (el mismo constructo). Este procedimiento de validación ha sido utilizado también por otros trabajos de educación histórica (Gestsdóttir, Van Boxtel \& Van Drie, 2018). El criterio establecido y señalado por diferentes autores es que un valor del alfa de Cronbach, entre $, 70 \mathrm{y}, 90$, indica una buena consistencia interna para una escala unidimensional (González y Pazmiño, 2015). En el caso del cuestionario se obtuvieron resultados adecuados: coeficiente alfa $=, 885$.

Igualmente se procedió a comprobar la validez del constructo y la viabilidad de un posterior análisis factorial. Para ello se analizó la prueba de esfericidad de Barlett y un Análisis de Componentes Principales (ACP) para cada una de las sub-escalas del cuestionario. Esta técnica explica el máximo porcentaje de varianza observada en cada ítem a partir de un número menor de 
componentes que resuma esa información (Lloret-Segura, Ferreres-Traver, Hernández-Baeza y Tomás-Marco, 2014).

En las dos sub-escalas se obtuvieron un nivel crítico (Sig.) de ,000 en la prueba de esfericidad de Barlett. Esto muestra que la matriz de datos es válida para continuar con el proceso de análisis factorial (tabla 1). Si aplicamos el ACP a cada uno de las sub-escalas, obtenemos una distribución en la primera de 4 dimensiones, explicando el $60,14 \%$ de la varianza total, con un $\mathrm{KMO}$ de ,82 (adecuado). En la segunda sub-escala obtenemos 7 dimensiones, explicando el 72,39\% de la varianza, con un KMO de ,89 (adecuado).

Los resultados obtenidos de estas pruebas mostraron que el cuestionario tiene un grado de fiabilidad y validez aceptables. Para este estudio se realizaron análisis de estadísticos descriptivos y se aplicaron pruebas de ANOVA para las variables de universidad de procedencia y formación previa, y pruebas no paramétricas (prueba de U-Mann Whitney) para la variable de género.

\section{Resultados}

\subsection{Recuerdos sobre la metodología didáctica en las clases de Historia}

En cuanto a los datos descriptivos, como se aprecia en las tablas 2 y 3 , los recuerdos sobre el uso de metodologías tradicionales en la clase de Geografía e Historia son superiores a los recuerdos de metodologías innovadoras. Como metodologías tradicionales se encontraban ítems como "El profesor o profesora utilizaba principalmente el método expositivo (lección magistral) para enseñar Historia" o "Mi principal papel como alumno/a era recoger apuntes y escuchar la lección". En el caso de metodologías innovadoras los ítems para valorar eran como "El profesor o profesora utilizaba con frecuencia fuentes históricas primarias" o "En el aula utilizábamos habitualmente la prensa y otros medios de comunicación para contextualizar o profundizar en temáticas históricas".

Igualmente, se ha realizado un análisis de varianza (ANOVA) para comprobar la hipótesis de que las medias de las universidades son iguales. Para ello se han agrupado de nuevo los ítems de recuerdos de metodología tradicional y los ítems de recuerdos de metodología innovadora. En los resultados se puede comprobar (tabla 4) que existe una diferencia significativa, aunque poco relevante (sig. ,042) entre las medias. Aplicando la prueba post-hoc de Tukey B se comprueba que los participantes de la Universidad de Málaga tienen una media significativamente menor en estos ítems de recuerdos de metodología tradicional con respecto a los participantes de la Universidad de Murcia. Ambos grupos de participantes se colocan en dos subconjuntos diferentes (tabla 4 y 5).

No se comprueba ninguna diferencia estadísticamente significativa entre los ítems agrupados sobre recuerdos de metodologías innovadoras en función de la universidad de procedencia (,287 en la prueba de ANOVA). Tampoco existen diferencias estadísticamente significativas entre los ítems agrupados sobre recuerdos de metodologías tradicionales e innovadoras en función de la formación previa de los participantes (,49 y ,69 en la prueba de ANOVA respectivamente).

Por su parte, las pruebas no paramétricas en función del género indican que la distribución de los Ítems agrupados sobre recuerdos de metodología tradicional en las clases de Historia es la misma en esta variable. Tanto las medias $(24,85$ en hombres y 25,18 en mujeres), y la prueba U de Mann-Whitney $(, 140)$ nos hacen confirmar la hipótesis nula (tabla 6). En esta línea, los resultados de las pruebas no paramétricas sobre la distribución de los ítems agrupados sobre recuerdos de metodología innovadora en las clases de Historia en función del género sí que muestran diferencias estadísticamente significativas. Así, tanto las medias
(19,17 para hombres y 16,28 para mujeres), y la prueba U de Mann-Whitney (,006), nos hacen afirmar la hipótesis alternativa. En este caso, los hombres tienen unos recuerdos sobre la metodología de las clases de Historia más innovadoras (tabla 7).

Por último, cabe señalar cómo existen correlaciones significativas $(-, 292)$ pero no muy grandes entre los ítems agrupados sobre los recuerdos del uso de metodologías tradicionales para la enseñanza de la Historia y los ítems agrupados sobre los recuerdos del uso de metodologías innovadoras para la enseñanza de la Historia. Los recuerdos de metodologías innovadoras o tradicionales funcionan como dos factores distintos. Pueden observarse participantes que recuerdan procesos innovadores, pero no por eso no recuerdan procesos tradicionales (tabla 8).

\subsection{Opiniones sobre la metodología didáctica en las clases de Historia}

El análisis de los datos descriptivos, como se aprecia en las tablas 9 y 10, revela cómo las opiniones sobre uso de metodologías innovadoras son superiores a las opiniones sobre el uso de metodologías tradicionales en la clase de Geografía e Historia. Las diferencias entre universidades no son muy amplias. En cuanto al uso de metodologías tradicionales, los participantes de la Universidad de Almería se posicionan en un extremo (más a favor) y los participantes de la Universidad de Murcia se posicional en el otro extremo (menos a favor). Esta disparidad se repite en las metodologías innovadoras, siendo los participantes de la Universidad de Almería los menos proclives a ellas y los de la Universidad de Murcia más favorables. Como metodologías y recursos tradicionales se encontraban ítems como el uso de la lección magistral o el uso del libro de texto. Como recursos más innovadores se encontraba el uso de fuentes o el uso de Apps y otras tecnologías emergentes.

Las pruebas de ANOVA por universidad no reflejan ninguna diferencia estadísticamente significativa entre los ítems agrupados sobre opiniones del profesorado acerca del uso de metodologías tradicionales o innovadores en el aula de Historia (,659 y ,207 en la prueba de ANOVA respectivamente). De la misma manera, tampoco existen diferencias estadísticamente significativas entre las opiniones del profesorado sobre el uso de metodologías tradicionales o innovadores en el aula de Historia en función de la formación previa de los participantes $(, 49$ y,21 en la prueba de ANOVA respectivamente, tabla 11).

Por su parte, las pruebas no paramétricas por género muestran diferencias estadísticamente significativas en la distribución de los ítems agrupados sobre la opinión del uso de metodologías tradicionales para la enseñanza de Historia. Las medias (15,83 para hombres y 16,05 para mujeres) y la prueba $U$ de Mann-Whitney $(, 88)$ nos permiten conservar la hipótesis nula (no hay diferencias estadísticamente significativas en función del género, tabla 12).

La distribución de los ítems agrupados sobre la opinión del uso de metodologías innovadores para la enseñanza de Historia en función del género sí muestra diferencias estadísticamente significativas. Las medias $(94,18$ para hombres y 97,18 para mujeres) y la prueba U de Mann-Whitney $(, 034)$ nos permiten afirmar la hipótesis alternativa (sí hay diferencias estadísticamente significativas en función del género, tabla 13).

Debido al gran número de ítems que agrupan las metodologías innovadoras, hemos dividido estos ítems en dos partes: a) variables que hacen referencia a técnicas y recursos de innovación relacionadas con el uso de las TIC y mass-media; y b) variables que hacen referencia a técnicas y recursos de innovación relacionadas con los procedimientos del historiador y estrategias metodológicas. 
La distribución de los ítems agrupados sobre la opinión del uso de metodologías innovadoras para la enseñanza de Historia relacionadas con el uso de las TIC y mass-media en función del género sí muestra diferencias estadísticamente significativas. Las medias (20,69 para hombres y 22,53 para mujeres) y la prueba U de Mann-Whitney (,006) nos permiten afirmar la hipótesis alternativa (sí hay diferencias estadísticamente significativas en función del género, tabla 14).

La distribución de los ítems agrupados sobre la opinión del uso de metodologías innovadoras relacionadas con el uso de procedimientos del historiador para la enseñanza de Historia en función del género no muestra diferencias estadísticamente significativas. Las medias (23,66 para hombres y 23,61 para mujeres) y la prueba U de Mann-Whitney $(326)$ nos permiten confirmar la hipótesis nula (no hay diferencias estadísticamente significativas en función del género, tabla 15).

Finalmente, existen correlaciones significativas $(-, 305)$ pero no muy grandes entre los ítems agrupados sobre percepción del uso de metodologías tradicionales para la enseñanza de la Historia y los ítems agrupados sobre percepción del uso de metodologías innovadoras para la enseñanza de la Historia (tabla 16).

\section{Discusión y conclusiones}

El análisis de los resultados nos permite establecer conclusiones en función de las valoraciones globales, las universidades, el género y la formación. En relación con los recuerdos, los ítems agrupados sobre metodología tradicional ofrecen unos resultados más elevados que los obtenidos en cuanto al uso de metodologías innovadoras. Los estudiantes reconocen que durante sus clases de Historia han aprendido fundamentalmente a través de estrategias y técnicas de naturaleza tradicional. Datos que coinciden con lo planteado en un buen número de trabajos (Martínez, Souto y Beltrán, 2006; Martínez, 2014; San Pedro, 2017; Gómez, Rodríguez y Mirete, 2018), en los que se constata el uso predominante de prácticas tradicionales por parte de los profesores de Educación Secundaria para la enseñanza de esta materia. El análisis de correlaciones muestra que los recuerdos de metodologías innovadoras o tradicionales se presentan como factores distintos y poco dependientes, lo que coincide con la diversidad y pluralidad del trabajo en las aulas (Martínez, Souto y Beltrán, 2006).

No se observan diferencias estadísticamente significativas en función de las universidades de procedencia y de la formación recibida, pero sí que existen en la comparación por género, en la que las metodologías innovadoras predominan en los recuerdos de los hombres. Esto puede explicarse por las diferentes concepciones que hombres y mujeres puedan tener sobre el significado de tradicional e innovador (Edwards-Schachters, 2018).

En cuanto a las opiniones sobre intenciones, los resultados muestran mayores puntuaciones en favor del uso de metodologías innovadoras, probablemente influidos por la tendencia actual que prima de introducir metodologías activas y aprendizajes integrados para favorecer el conocimiento social y sus finalidades (Gómez, Ortuño y Miralles, 2018). Estos resultados coindicen con los aportados por Montilla, Maraver, Romero y Martín (2018), quienes, en un reciente trabajo dedicado al análisis de las expectativas del profesorado novel en formación, señalan, como una de sus principales inquietudes, la inclusión en las aulas de recursos y estrategias que aseguren la motivación, así como el aprendizaje activo y significativo del alumnado. No se observan diferencias estadísticas en función de la universidad de procedencia ni de la formación previa de los participantes, y sí en lo relativo al género. En este caso, las mujeres tienen una opinión más favorable hacia el uso de metodologías innovadoras para la enseñanza de la Historia, especialmente en los ítems relacionados con el uso de las TIC y los mass-media. Estos resultados coinciden con los presentados en las últimas investigaciones, en las que se comienza a evidenciar la ausencia de una brecha digital de género (Cózar y Roblizo, 2014; Roblizo y Cózar, 2015). De este modo, pese a que los hombres parecen tener mayores habilidades técnicas, más experiencia y conocimiento en estas tecnologías, las mujeres destacarían por estar más interesadas en usarlas para el aprendizaje y en su propia formación.

Como apuntamos en el marco teórico, el debate en torno a cómo influye el género en las percepciones y actitudes que tienen hombres y mujeres frente a las TIC ha estado presente desde hace décadas. En este sentido, mientras autores como Ory (1997) afirmaban que las diferencias existentes eran menores, otros como Merrill (1991) sugerían que los hombres se sentían más seguros que las mujeres con las tecnologías. Estudios más recientes, como el de Admiraal et al. (2017), han arrojado resultados similares. Sin embargo, en contraposición a estas posturas, existen investigaciones que no encuentran diferencias significativas en cuanto al género (Shaw y Marlow, 1999).

Una tercera vertiente es la que muestra esta investigación, donde observamos que las mujeres presentan una actitud más favorable que los hombres hacia la implementación de recursos digitales en el aula. Los resultados obtenidos revelan la necesidad de ahondar en una línea de investigación que profundice en el género como posible variable que influye en la valoración y uso de las nuevas tecnologías en los contextos educativos. En caso de confirmarse el género como factor que predispone a unas actitudes u otras frente a las TIC, este hecho podría tener implicaciones en las estrategias metodológicas y recursos docentes que emplean los profesores y profesoras en sus aulas.

Para finalizar, a modo de conclusión, creemos que es necesaria una intervención en los programas de formación del profesorado que permita reforzar una educación histórica basada en competencias mediante la implementación de metodologías activas de aprendizaje y el uso de estrategias y enfoques innovadores. Sabemos que las concepciones y experiencias escolares previas tienen un peso muy importante en las prácticas docentes que desarrollará el futuro profesorado de las distintas etapas educativas (González y Fuentes, 2011). Como señala Estepa (2017: 26), los principales referentes que guían los procesos de enseñanza de los y las docentes son sus propios recuerdos que conservan de su época de estudiantes. Por eso es tan importante conocer con qué concepciones epistemológicas y metodológicas viene nuestro alumnado, para actuar sobre ellas y transformarlas.

\section{Referencias bibliográficas}

Admiraal, W.; Louws, M.; Lockhorst, D.; Paas, T.; Buynsters, M.; Cviko, A.; Janssen, C.; De Jonge, M.; Nouwens, S.; Post, L.; Van der Ven, F. y Kester, L. (2017). Teachers in school-based technology innovations: A typology of their beliefs on teaching and technology. Computers $\mathcal{E}$ Education, 114, 57-68.

Angelini, M. L. y García-Carbonell, A. (2015). Percepciones sobre la integración de modelos pedagógicos en la formación del profesorado: la simulación y juego y el flipped classroom. Education in the Knowledge Society, 16(2), 16-30.

Arancibia, M. M. y Badia, A. (2015). Concepciones de profesores de secundaria sobre enseñar y aprender Historia con TIC. Revista Electrónica de Investigación Educativa, 17(2), 62-76.

Barba, J. J., Martínez, S. y Torrego, L. (2012). El proyecto de aprendizaje tutorado cooperativo. Una experiencia en el grado de maestra de Educación Infantil. Revista de Docencia Universitaria, 10(1), 123144. DOI: https://doi.org/10.4995/redu.2012.6125 
Blasco-Serrano, A. C., Lorenzo, J. y Sarsa, J. (2018). Percepción de los estudiantes al invertir la clase mediante el uso de redes sociales y sistemas de respuesta inmediata. RED. Revista de Educación a Distancia, 57(6), 1-19. DOI: http://dx.doi. org $/ 10.6018 / \mathrm{red} / 57 / 6$

Cabero, J. y Guerra, S. (2011). La alfabetización y formación en medios de comunicación en la formación inicial del profesorado. Educación XX1, 14(1), 89-115.

Cabero, J., Barroso, J., Llorente, M. C. y Yanes, C. (2016). Redes sociales y Tecnologías de la Información y la Comunicación en Educación: aprendizaje colaborativo, diferencias de género, edad y preferencias. RED. Revista de Educación a Distancia, 51, 1-23. DOI: http:/ /dx.doi.org/10.6018/red/51/1

Cabezas, M., Casillas, S. y Pinto, A. M. (2014). Percepción de los alumnos de Educación Primaria de la universidad de Salamanca sobre su competencia digital. EDUTEC. Revista Electrónica de Tecnología Educativa, 48, 1-14. DOI: https://doi. org/10.21556/edutec.2014.48.156

Castaño, C., Martín, J. y Martínez, J. L. (2011). La brecha digital de género en España y Europa: medición con indicadores compuestos. Reis. Revista Española de Investigaciones Sociológicas, 136, 127-140. DOI: $10.5477 /$ cis / reis.136.127

Castro, L. y López, R. (2019). Educación patrimonial: necesidades sentidas por el profesorado de infantil, primaria y secundaria. Revista Interuniversitaria de Formación del Profesorado, 94(33.1), 97-114.

Colomer, J. C.; Saiz, J. y Bel, J. C. (2018). Competencia digital en futuros docentes de Ciencias Sociales en Educación Primaria: análisis desde el modelo TPACK. Educatio Siglo XXI, vol. 36(1), 107-128.

Cózar, R. y Roblizo, M. (2014). La competencia digital en la formación de los futuros maestros. Percepciones de los alumnos de los Grados de Maestros de la Facultad de Educación de Albacete. RELATEC. Revista Latinoamérica de Tecnología Educativa, 13(2), 119-133.

Cózar, R., Moya, M. V., Hernández, J. A. y Hernández, J. R. (2016). Cocimiento y Uso de las Tecnologías de la Información y las Comunicaciones (TIC) según el estilo de Aprendizaje de los Futuros Maestros. Formación Universitaria, 9(6), 105-118.

Cózar, R., González-Calero, J. A., Villena, R. y Merino, J. M. (2019). Análisis de la motivación ante el uso de la realidad virtual en la enseñanza de la historia en futuros maestros. EDUTEC. Revista Electrónica de Tecnología Educativa, 68, 1-14. DOI: https://doi.org/10.21556/edutec.2019.68.1315

Cuenca, J. M., Estepa, J., y Martín, M. (2016). Patrimonio, educación, identidad y ciudadanía. Profesorado y libros de texto en la enseñanza obligatoria. Revista de Educación, 375, 136-159.

De Haro, J.J. (2009). Algunas experiencias de innovación educativa. ARBOR. Ciencia, pensamiento y cultura, CLXXXV, 71-92.

Edwards-Schachters, M. (2018). The nature and variety of innovation. International Journal of Innovation Studies, 2, 65-79. DOI: $10.1016 /$ j.ijis.2018.08.004

Estepa, J. (2001). El patrimonio en la didáctica de las Ciencias Sociales: obstáculos y propuestas para su tratamiento en el aula. Íber, 30, 93-106.

Estepa, J. (2017). Otra didáctica de la Historia para otra escuela. Huelva: Universidad de Huelva.

Estepa, J.; Ávila, R. y Ferreras, M. (2008). Primary and secondary teachers' conceptions about heritage and heritage education: A comparative analysis. Teaching and Teacher Education, 26, 2095-2107.

Fontal, O. e Ibáñez, Á. (2017). La investigación en Educación Patrimonial. Evolución y estado actual a través del análisis de indicadores de alto impacto. Revista de Educación, n ${ }^{\circ}$ 375, 184-214.

Gestsdóttir, S. M., Van Boxtel, C., Van Drie, J. (2018). Teaching historical thinking and reasoning: Construction of an obser- vation instrument. British Educational Research Journal, 44 (6), 960-981. DOI: 10.1002/berj.3471

Gómez,C.J.y Miralles,P.(2015).¿Pensarhistóricamente omemorizar el pasado? La evaluación de los contenidos históricos en la educación obligatoria en España. Revista de Estudios Sociales, 52, 52-68. DOI: http:/ /dx.doi.org/10.7440/res52.2015.04

Gómez, C. J., Ortuño, J. y Miralles, J. (2018). Enseñar Ciencias Sociales con métodos activos de aprendizaje. Reflexiones y propuestas a través de la indagación. Barcelona: Octaedro.

Gómez, C. J., Rodríguez, R. A. y Mirete, A. B. (2018). Percepción de la enseñanza de la historia y concepciones epistemológicas. Una investigación con futuros maestros. Revista Complutense de Educación, 29(1), 237-250. DOI: https://doi.org/10.5209/ RCED.52233

González, J. y Pazmiño, M. (2015). Cálculo e interpretación del Alfa de Cronbach para el caso de validación de la consistencia interna de un cuestionario, con dos posibles escalas tipo Likert. Revista Publicando, 2(1), 62-77.

González-Peiteado; M. y Pino-Juste, M. (2013). Percepción del alumnado de Ciencias de la Educación de la Universidad de Santiago de Compostela sobre el uso de los estilos de enseñanza. Innovación Educativa, 23, 215-229.

González-Peiteado; M. y Pino-Juste, M. (2014). Aproximación a las representaciones y creencias del alumnado de Magisterio sobre los estilos de enseñanza. Educación XXI, 17(1), 83-110.

González, M. y Fuentes, E. J. (2011). El Practicum en el aprendizaje de la profesión docente. Revista de Educación, 354, 47-70.

Gutiérrez, A., Palacios, A. y Torrego, L. (2010). La formación de los futuros maestros y la integración de las TIC en la educación: anatomía de un desencuentro. Revista de Educación, 352, 267-293.

Jiménez, R.; Cuenca, J. Mª y Ferreras, M. (2010). Heritage education: Exploring the conceptions of teachers and administrators from the perspective of experimental and social science. Teaching and Teacher Education, 26, 1319 - 1331.

Lévesque, S. y Zanazanian, P. (2015). History Is A Verb: "We Learn It Best When We Are Doing It!": French and English Canadian Prospective Teachers and History. Revista de Estudios Sociales, 52, 32-51.

Levstik, L. y Barton, K. C. (2001). Doing History. Investigating with Children in Elementary and Middle Schools. Second Edition. Mahwah. New Jersey: Lawrence Erlbaum Associates (LEA).

Lloret-Segura, S., Ferreres-Traver, A., Hernández-Baeza, A., y Tomás-Marco, I. (2014). Exploratory Item Factor Analysis: A practical guide revised and up-dated. Annals of Psycholo$g y, 30(3), 1151-1169$.

Marqués, P. (2013). Impacto de las TIC en la educación: funciones y limitaciones. 3C TIC: cuadernos de desarrollo aplicados a las TIC, 2(1), 2-15.

Martínez, N. (Coord.) (2014). La historia de España en los recuerdos escolares: análisis, interpretación y poder de cambio de los testimonios de profesores y alumnos. Valencia: Nau Llibres.

Martínez, N., Souto, X. M. y Beltrán, J. (2006). Los profesores de historia y la enseñanza de la historia en España. Una investigación a partir de los recuerdos de los alumnos. Enseñanza de las Ciencias Sociales: Revista de Investigación, 5, 55-71.

Merchán, F. J. (2011). El control de la conducta del alumnado en el Aula. ¿Un problema para la práctica de la investigación escolar? Investigación en la escuela, 73, 53-64.

Merrill, M. D. (1991). Constructivism and instructional design. Educational Technology, 31(5), 45-53.

Miralles, P. y Gómez, C. J. (2016). Sin «Cronos» ni «Kairós». El tiempo histórico en los exámenes de $1^{\circ}$ y $2^{\circ}$ de Educación Secundaria Obligatoria. Enseñanza de las Ciencias Sociales, 15, 15-26. DOI: 10.1344/ECCSS2016.15.2 
Miralles, P.; Gómez, C. J. y Rodríguez, R. (2017). Patrimonio, competencias históricas y metodologías activas de aprendizaje. Un análisis de las opiniones de los docentes en formación en España e Inglaterra. Estudios Pedagógicos, 4, 161-184.

Miralles, P.; Gómez, C. J. y Monteagudo, J. (2019). Percepciones sobre el uso de recursos TIC y «mass-media» para la enseñanza de la historia. Un estudio comparativo en futuros docentes de España-Inglaterra. Educación XX1, 22(2), 187-211, doi:10.5944/educXX1.21377

Miralles, P., Gómez, C. J., Arias, V. B. y Fontal, O. (2019). Recursos digitales y metodología didáctica en la formación inicial de docentes de Historia. Comunicar, 61, 45-56. https://doi. org/10.3916/C61-2019-04

Montilla, M. V. C., Maraver, P., Romero, C. y Martín, A. (2018). Análisis de las expectativas del profesorado novel sobre su futura labor docente. Aula Abierta, 47(2), 185-192.

Moya, M. V., Hernández, J. R., Hernández, J. A. y Cózar, R. (2011). Análisis de los estilos de aprendizaje y las TIC en la formación personal del alumnado universitario a través del cuestionario REATIC. Revista de Investigación Educativa, 29(1), 137-156.

Ory, J. (1997). Gender similarity in the use of and attitudes about ALN in a university setting. Journal of Asynchronous Learning Networks, 1(1). (http://www.aln.org/alnweb/journal/issue1/ory.htm)

Parra, D. y Fuertes, C. (Coords.) (2019). Reinterpretar la tradición, transformar las prácticas. Ciencias Sociales para una educación crítica. Valencia: Tirant humanidades.

Pegalajar, M. C. y Colmenero, M. J. (2013). Percepciones hacia el aprendizaje cooperativo en estudiantes del Grado de Maestro. Revista de Docencia Universitaria, 11(3), 342-362.

Prats, J. (2010). En defensa de la historia como materia educativa. Tejuelo. Didáctica de la Lengua y la Literatura, 9, 8-17.

Ramírez-García, A. y González-Fernández, N. (2016). Competencia mediática del profesorado y del alumnado de educación obligatoria en España/Media Competence of Teachers and Students of Compulsory Education in Spain. Comunicar, 24(49), 49-58.
Roblizo, M. y Cózar, R. (2015). Usos y competencias en TIC en los futuros maestros de educación infantil y primaria: hacia una alfabetización tecnológica real para docentes. Píxel-Bit. Revista de Medios y Educación, 47, 23-39.

Sáiz, J. y Gómez, C. J. (2016). Investigar el pensamiento histórico y narrativo en la formación del profesorado: fundamentos teóricos y metodológicos. Revista electrónica interuniversitaria de formación del profesorado, 19(1), 175-190. DOI: http:/ / dx.doi. org/10.6018/reifop.19.1.206701

Sáiz, J. y Muñoz, A. (2014). Las opiniones del profesorado: representaciones sociales y biográficas. En N. Martínez Valcárcel (Coord.), La historia de España en los recuerdos escolares. Análisis, interpretación y poder de cambio de los testimonios de profesores y alumnos (pp. 211-226). Valencia: Nau Llibres.

San Pedro, M. B. y López, I. (2017). El profesorado de Ciencias Sociales en contextos rurales de Asturias: concepciones y uso de recursos educativos. Aula Abierta, 45, 33-40. DOI: http:// dx.doi.org/10.17811/rifie.45.2017.33-40

Sapsford, R. y Jupp V. (2006). Data Collection and Analysis. London: Sage Publications.

Shaw, G. y Marlow, N. (1999). The role of student learning styles, gender, attitudes and perceptions on information and communication technology assisted learning. Computers $\mathcal{E}$ Education, 33, 223-234.

Spennemann, D. R. (1996). Gender imbalances in computer access among environmental science students. Journal of Instructional Science and Technology, 1(2). (www.usq.edu.au/ electpub/e-jist/spenne.htm)

Suárez, M. Á. (2012). Concepciones sobre la Historia en Primaria: La epistemología como asunto clave en la formación inicial de maestros. Didáctica de las Ciencias Experimentales y Sociales, 26, 73-93.

VanSledright, B. A. (2014). Assessing Historical Thinking and Understanding. Innovation Design for New Standards. New York: Routledge.

Tabla 1.

Prueba de esfericidad de Barlett y KMO de los bloques del cuestionario

\begin{tabular}{lccc}
\hline Sub-escala & $\begin{array}{c}\text { Sig. En la prueba de } \\
\text { esfericidad de Barlett }\end{array}$ & $\begin{array}{c}\text { KMO. Medida Kaiser-Meyer- } \\
\text { Olkin de adecuación de } \\
\text { muestreo }\end{array}$ & Número de dimensiones \\
\hline $\begin{array}{l}\text { Sub-escala de recuerdos } \\
\text { de metodología }\end{array}$ &, 000 &, 82 & 4 \\
$\begin{array}{l}\text { Sub-escala de opiniones } \\
\text { sobre metodología }\end{array}$ &, 000 &, 89 & 7 \\
\hline
\end{tabular}

Tabla 2.

Estadísticos descriptivos los ítems agrupados sobre recuerdos de metodología tradicional en el aula de Geografía e Historia

\begin{tabular}{|c|c|c|c|c|c|c|}
\hline \multirow{2}{*}{ Universidad } & \multirow{2}{*}{$\mathrm{N}$} & \multirow{2}{*}{ Media } & \multirow{2}{*}{$\begin{array}{l}\text { Desviación } \\
\text { estándar }\end{array}$} & \multicolumn{2}{|c|}{$95 \%$ del intervalo de confianza para la media } & \multirow{2}{*}{ Mínimo-Máximo } \\
\hline & & & & Límite inferior & Límite superior & \\
\hline Universidad de Málaga & 35 & 24,0 & 3,42 & 22,82 & 25,17 & $12,00-29,00$ \\
\hline Universidad de Castilla-La Mancha & 32 & 25,09 & 2,65 & 24,13 & 26,05 & $18,00-30,00$ \\
\hline Universidad de Almería & 26 & 25,07 & 2,44 & 24,08 & 26,06 & $18,00-28,00$ \\
\hline Universidad de Murcia & 35 & 25,82 & 1,77 & 25,21 & 26,43 & $21,00-29,00$ \\
\hline Total & 128 & 24,99 & 2,71 & 24,51 & 25,46 & $12,00-30,00$ \\
\hline
\end{tabular}


Tabla 3.

Estadísticos descriptivos de los ítems agrupados sobre recuerdos de metodología innovadora en el aula de Geografía e Historia

\begin{tabular}{|c|c|c|c|c|c|c|}
\hline & \multirow{2}{*}{$\mathrm{N}$} & \multirow{2}{*}{ Media } & \multirow{2}{*}{$\begin{array}{l}\text { Desviación } \\
\text { estándar }\end{array}$} & \multicolumn{2}{|c|}{$95 \%$ del intervalo de confianza para la media } & \multirow{2}{*}{ Mínimo- Máximo } \\
\hline & & & & Límite inferior & Límite superior & \\
\hline Universidad de Málaga & 35 & 17,37 & 6,33 & 15,19 & 19,54 & $9,00-38,00$ \\
\hline Universidad de Castilla-La Mancha & 33 & 19,30 & 5,95 & 17,19 & 21,41 & $9,00-30,00$ \\
\hline Universidad de Almería & 26 & 18,34 & 5,59 & 16,08 & 20,60 & $11,00-35,00$ \\
\hline Universidad de Murcia & 35 & 16,65 & 5,69 & 14,69 & 18,61 & $10,00-32,00$ \\
\hline Total & 129 & 17,86 & 5,94 & 16,83 & 18,90 & $9,00-38,00$ \\
\hline
\end{tabular}

Tabla 4.

Análisis de varianza ANOVA de los ítems agrupados sobre recuerdos de metodologías tradicionales según universidad de procedencia de los participantes

\begin{tabular}{lccccc}
\hline & $\begin{array}{c}\text { Suma de } \\
\text { cuadrados }\end{array}$ & gl & $\begin{array}{c}\text { Media } \\
\text { cuadrática }\end{array}$ & F & Sig. \\
\hline Entre grupos & 59,456 & 3 & 19,819 & 2,807 &, 042 \\
$\begin{array}{l}\text { Dentro de } \\
\text { grupos }\end{array}$ & 875,536 & 124 & 7,061 & & \\
Total & 934,992 & 127 & & & \\
\hline
\end{tabular}

Tabla 6.

Datos de medias según género sobre recuerdos de metodología tradicional

\begin{tabular}{lcc}
\hline & $\begin{array}{c}\text { Sexo } \\
\text { Hombre } \\
\text { Media }\end{array}$ & $\begin{array}{c}\text { Mujer } \\
\text { Media }\end{array}$ \\
\hline $\begin{array}{l}\text { Ítems agrupados sobre recuerdos } \\
\text { de metodología tradicional en las } \\
\text { clases de Geografía e Historia }\end{array}$ & $24,85_{a}$ & $25,18_{a}$ \\
\hline
\end{tabular}

Tabla 5.

Prueba post-hoc de Tukey B de los ítems agrupados sobre recuerdos de metodologías tradicionales según universidad de procedencia de los participantes

\begin{tabular}{|c|c|c|c|c|}
\hline & \multirow[t]{2}{*}{ Universidad } & \multirow[t]{2}{*}{$\mathrm{N}$} & \multicolumn{2}{|c|}{$\begin{array}{c}\text { Subconjunto para } \\
\text { alfa }=0.05\end{array}$} \\
\hline & & & 1 & 2 \\
\hline \multirow{4}{*}{ Tukey B ${ }^{a, b}$} & Universidad de Málaga & 35 & 24,0000 & \\
\hline & Universidad de Almería & 26 & 25,0769 & 25,0769 \\
\hline & $\begin{array}{l}\text { Universidad de Castilla-La } \\
\text { Mancha }\end{array}$ & 32 & 25,0938 & 25,0938 \\
\hline & Universidad de Murcia & 35 & & 25,8286 \\
\hline \multicolumn{5}{|c|}{$\begin{array}{l}\text { Se visualizan las medias para los grupos en los subconjuntos homogéneos. } \\
\text { a. Utiliza el tamaño de la muestra de la media armónica }=31,532 \text {. } \\
\text { b. Los tamaños de grupo no son iguales. Se utiliza la media armónica } \\
\text { de los tamaños de grupo. Los niveles de error de tipo I no están } \\
\text { garantizados. }\end{array}$} \\
\hline
\end{tabular}

Tabla 7.

Datos de medias según género sobre recuerdos de metodología innovadora

\begin{tabular}{lcc}
\hline & $\begin{array}{c}\text { Hombre } \\
\text { Media }\end{array}$ & $\begin{array}{c}\text { Mujer } \\
\text { Media }\end{array}$ \\
\hline $\begin{array}{l}\text { Ítem agrupados sobre } \\
\text { recuerdos de metodología } \\
\text { innovadora en las clases de } \\
\text { Geografía e Historia }\end{array}$ & $19,17_{\mathrm{a}}$ & $16,28_{\mathrm{b}}$ \\
\hline
\end{tabular}

Tabla 8.

Correlaciones entre items agrupados sobre recuerdos de metodología tradicional e innovadora

Ítems agrupados sobre recuerdos del uso de metodologías

tradicionales para la enseñanza de Historia.
Ítems agrupados sobre recuerdos del uso de metodologías innovadoras para la enseñanza de Historia

Ítems agrupados sobre recuerdos del uso de metodologías tradicionales para la enseñanza de Historia.

Correlación de Pearson

Sig. (bilateral)
1

\section{$-, 292^{*}$}

,001

128

128
Ítems agrupados sobre recuerdos del uso de metodologías innovadoras para la enseñanza de Historia.

Correlación de Pearson
Sig. (bilateral)

$\mathrm{N}$

,$- 292^{* *}$
, 001
128

128
1

**. La correlación es significativa en el nivel 0,01 (2 colas). 
Tabla 9.

Estadísticos descriptivos de los ítems agrupados sobre la percepción del uso de metodologías tradicionales para la enseñanza de la Historia

\begin{tabular}{|c|c|c|c|c|c|c|}
\hline & \multirow{2}{*}{$\mathrm{N}$} & \multirow{2}{*}{ Media } & \multirow{2}{*}{$\begin{array}{l}\text { Desviación } \\
\text { estándar }\end{array}$} & \multicolumn{2}{|c|}{$95 \%$ del intervalo de confianza para la media } & \multirow{2}{*}{ Mínimo-Máximo } \\
\hline & & & & Límite inferior & Límite superior & \\
\hline Universidad de Málaga & 35 & 16,22 & 3,81 & 14,91 & 17,53 & $9,00-24,00$ \\
\hline $\begin{array}{l}\text { Universidad de Castilla-La } \\
\text { Mancha }\end{array}$ & 33 & 16,06 & 2,98 & 15,00 & 17,12 & $9,00-21,00$ \\
\hline Universidad de Almería & 25 & 16,40 & 3,21 & 15,07 & 17,72 & $11,00-23,00$ \\
\hline Universidad de Murcia & 34 & 15,14 & 3,87 & 13,79 & 16,49 & $6,00-22,00$ \\
\hline Total & 127 & 15,92 & 3,51 & 15,31 & 16,54 & $6,00-24,00$ \\
\hline
\end{tabular}

Tabla 10.

Estadísticos descriptivos de los ítems agrupados sobre la percepción del uso de metodologías innovadoras para la enseñanza de la Historia

\begin{tabular}{|c|c|c|c|c|c|c|}
\hline & \multirow{2}{*}{$\mathrm{N}$} & \multirow{2}{*}{ Media } & \multirow{2}{*}{$\begin{array}{l}\text { Desviación } \\
\text { estándar }\end{array}$} & \multicolumn{2}{|c|}{$95 \%$ del intervalo de confianza para la media } & \multirow{2}{*}{ Mínimo-Máximo } \\
\hline & & & & Límite inferior & Límite superior & \\
\hline Universidad de Málaga & 34 & 94,26 & 23,06 & 86,21 & 102,31 & $31,00-121,00$ \\
\hline $\begin{array}{l}\text { Universidad de Castilla-La } \\
\text { Mancha }\end{array}$ & 31 & 98,54 & 13,52 & 93,58 & 103,50 & $72,00-120,00$ \\
\hline Universidad de Almería & 26 & 89,53 & 21,32 & 80,92 & 98,15 & $38,00-118,00$ \\
\hline Universidad de Murcia & 32 & 98,78 & 15,57 & 93,16 & 104,39 & $43,00-124,00$ \\
\hline Total & 123 & 95,52 & 18,89 & 92,14 & 98,89 & $31,00-124,00$ \\
\hline
\end{tabular}

Tabla 11.

Pruebas de ANOVA sobre percepción del uso de metodologías tradicionales o innovadoras en la clase de Historia en función de la Universidad y formación previa

Percepción sobre el uso de metodologías tradicionales en función de Universidad
Percepción sobre el uso de metodologías innovadoras en función de Universidad
Percepción sobre el uso de metodologías tradicionales en función de la formación previa
Percepción sobre el uso de metodologías innovadoras en función de la formación previa

Prueba de ANOVA

, 659

,207

, 49

,21

Tabla 12.

Resultados de medias en función del género sobre el uso de metodologías tradicionales

\begin{tabular}{lcc}
\hline & \multicolumn{2}{c}{ Sexo } \\
& $\begin{array}{l}\text { Hombre } \\
\text { Media }\end{array}$ & $\begin{array}{c}\text { Mujer } \\
\text { Media }\end{array}$ \\
\hline $\begin{array}{l}\text { Media de los ítems agrupados sobre } \\
\text { la percepción del uso de metodologías } \\
\text { tradicionales para la enseñanza de la }\end{array}$ & $15,83_{\mathrm{a}}$ & $16,05_{\mathrm{a}}$ \\
\begin{tabular}{l} 
Historia \\
\hline
\end{tabular} & & \\
\hline
\end{tabular}

Tabla 13.

Resultados de medias en función del género sobre el uso de metodologías innovadoras

\begin{tabular}{lcc}
\hline & \multicolumn{2}{c}{ Sexo } \\
& $\begin{array}{c}\text { Hombre } \\
\text { Media }\end{array}$ & $\begin{array}{c}\text { Mujer } \\
\text { Media }\end{array}$ \\
\hline $\begin{array}{l}\text { Media de los ítems agrupados sobre la per- } \\
\text { cepción del uso de metodologías innovado- } \\
\text { ras para la enseñanza de la Historia }\end{array}$ & 94,18 & 97,18 \\
\hline
\end{tabular}


Tabla 14

Resultados de medias en función del género sobre el uso de metodologías innovadoras ligadas a recursos digitales

\begin{tabular}{lcc}
\hline & $\begin{array}{c}\text { Sexo } \\
\text { Hombre } \\
\text { Media }\end{array}$ & $\begin{array}{c}\text { Mujer } \\
\text { Media }\end{array}$ \\
\hline & & \\
$\begin{array}{l}\text { Media de los ítems agrupados sobre la per- } \\
\text { cepción del uso de metodologías innova- } \\
\text { doras ligadas a recursos digitales }\end{array}$ & 20,69 & 22,53 \\
\hline
\end{tabular}

Tabla 15.

Resultados de medias en función del género sobre el uso de metodologías innovadoras ligadas a procedimientos (uso de fuentes)

\begin{tabular}{lcc}
\hline & $\begin{array}{c}\text { Hombre } \\
\text { Media }\end{array}$ & $\begin{array}{c}\text { Mujer } \\
\text { Media }\end{array}$ \\
\hline $\begin{array}{l}\text { Media de los ítems agrupados sobre } \\
\text { la percepción del uso de metodolo- } \\
\text { gías innovadoras ligadas al uso de } \\
\text { procedimientos para la enseñanza de } \\
\text { la Historia }\end{array}$ & 23,66 & \\
\hline
\end{tabular}

Tabla 16.

Correlaciones de Pearson entre opiniones del uso de metodologías tradicionales e innovadoras

\section{Correlaciones}

Ítems agrupados sobre percepción del uso de metodologías tradicionales para la enseñanza de la Historia.
Ítems agrupados sobre percepción del uso de metodologías innovadoras para la enseñanza de la Historia.

\begin{tabular}{|c|c|c|c|}
\hline $\begin{array}{l}\text { Ítems agrupados sobre } \\
\text { percepción del uso de }\end{array}$ & Correlación de Pearson & 1 &,$- 305^{* *}$ \\
\hline $\begin{array}{l}\text { metodologías tradicionales } \\
\text { para la enseñanza de la }\end{array}$ & Sig. (bilateral) & & ,001 \\
\hline Historia. & $\mathrm{N}$ & 127 & 122 \\
\hline $\begin{array}{l}\text { Ítems agrupados sobre } \\
\text { percepción del uso de }\end{array}$ & Correlación de Pearson &,$- 305^{* *}$ & 1 \\
\hline $\begin{array}{l}\text { metodologías innovadoras } \\
\text { para la enseñanza de la }\end{array}$ & Sig. (bilateral) & ,001 & \\
\hline Historia. & $\mathrm{N}$ & 122 & 123 \\
\hline
\end{tabular}

